\title{
Correction to: Role of anemia and proteinuria in the development of subsequent renal function deterioration in a general population with preserved glomerular filtration rate: a community-based cohort study
}

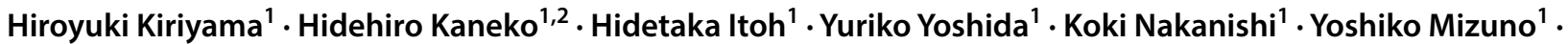 \\ Masao Daimon ${ }^{1,3} \cdot$ Hiroyuki Morita $^{1} \cdot$ Yutaka Yatomi $^{3} \cdot$ Issei Komuro $^{1}$
}

Published online: 19 August 2019

(C) Italian Society of Nephrology 2019

\section{Correction to: Journal of Nephrology https://doi.org/10.1007/s40620-019-00605-2}

After the online first publication of their research, the authors realized they made several mistakes in the data conversion process from the original data of health checkup at the Center for Preventive Medicine at The University of Tokyo Hospital to the datasheet which was used for the statistical analysis by SPSS. Particularly, the prevalence of hypertension, diabetes mellitus, and hypercholesterolemia was not appropriately analyzed. Therefore, the authors reanalyzed the data according to the corrected database.

Methods and results in the Abstract should read as it follows:

Among 3217 subjects who underwent repeated health check-ups, we excluded 478 subjects with eGFR $<60 \mathrm{~mL} /$ $\mathrm{min} / 1.73 \mathrm{~m}^{2}$ and examined 2739 subjects. EGFR decline rate was calculated from the difference in eGFR between the first and last visits. EGFR decline, which was defined as a drop in GFR accompanied by a $25 \%$ or greater drop in eGFR from baseline and/or a sustained decline of more than $5 \mathrm{~mL} /$ $\mathrm{min} / 1.73 \mathrm{~m}^{2} /$ year, was observed in 209 subjects (7.6\%).

The original article can be found online at https://doi.org/10.1007/ s40620-019-00605-2.

Hidehiro Kaneko

kanekohidehiro@gmail.com

1 The Department of Cardiovascular Medicine, The University of Tokyo, Tokyo, Japan

2 The Department of Advanced Cardiology, The University of Tokyo, 7-3-1, Hongo, Bunkyo-ku, Tokyo 113-8655, Japan

3 The Department of Clinical Laboratory, The University of Tokyo Hospital, Tokyo, Japan
Anemia according to the WHO definition (16.7\% vs. $11.7 \%, \mathrm{p}=0.03)$, and proteinuria $(3.3 \%$ vs. $0.8 \%, \mathrm{p}=0.001)$ at baseline were more commonly observed in subjects with eGFR decline. Multivariable logistic regression analysis showed that anemia and proteinuria were independently associated with eGFR decline.

In the "Methods" section the sub-heading "Measurement of covariates" should begin with

At baseline, general information and prior medical history were recorded by a standard interview. Body mass index was calculated in $\mathrm{kg} / \mathrm{m}^{2}$. Hypertension was defined as a blood pressure $\geq 140 / 90 \mathrm{mmHg}$ or use of antihypertensive medications. Diabetes mellitus (DM) was defined by fasting glucose $\geq 126 \mathrm{mg} / \mathrm{dL}$ or use of insulin or oral antidiabetic medications. Hypercholesterolemia was defined by total cholesterol $>240 \mathrm{mg} / \mathrm{dL}$ or use of antihypercholesterolemic medications.

In the "Results" section under the sub-heading "Baseline characteristics"

Anemia was more common in the eGFR decline group than in the eGFR non-decline group $(11.7 \%$ vs. $16.7 \%$; $\mathrm{p}=0.03)$. EGFR at baseline was significantly higher in the eGFR decline group $\left(76.9 \pm 11.7 \mathrm{~mL} / \mathrm{min} / 1.73 \mathrm{~m}^{2}\right.$ vs. $\left.85.4 \pm 21.4 \mathrm{~mL} / \mathrm{min} / 1.73 \mathrm{~m}^{2} ; \mathrm{p}<0.001\right)$, eGFR at the last visit was significantly higher in the eGFR non-decline group $\left(75.2 \pm 12.6 \mathrm{~mL} / \mathrm{min} / 1.73 \mathrm{~m}^{2}\right.$ vs. $71.5 \pm 20.4 \mathrm{~mL} /$ $\min / 1.73 \mathrm{~m}^{2} ; \mathrm{p}=0.011$ )

the last sentence should read as it follows:

Proteinuria was more commonly observed in the eGFR decline group $(3.3 \%$ vs. $0.8 \%, p=0.001)$.

In the "Results" section under the sub-heading "Determinants of eGFR decline"

Multivariable logistic regression analysis including gender, age ( $\geq 60$ years old), body mass index $(>25 \mathrm{~kg} /$ 
Table 1 Baseline clinical characteristics

\begin{tabular}{|c|c|c|c|c|}
\hline Variable & Overall $(n=2739)$ & $\begin{array}{l}\text { eGFR non-decline group } \\
(\mathrm{n}=2530)\end{array}$ & $\begin{array}{l}\text { eGFR decline group } \\
(\mathrm{n}=209)\end{array}$ & $\mathrm{p}$ value \\
\hline Gender (male) & $1519(55.5)$ & $1410(55.7)$ & $109(52.2)$ & 0.32 \\
\hline Age (year) & $58.9 \pm 11.8$ & $58.9 \pm 11.8$ & $58.5 \pm 11.5$ & 0.67 \\
\hline Follow-up period (day) & $1233 \pm 494$ & $1278 \pm 472$ & $686 \pm 414$ & $<0.001$ \\
\hline BMI & $22.9 \pm 3.4$ & $22.9 \pm 3.4$ & $22.6 \pm 3.2$ & 0.16 \\
\hline Systolic blood pressure $(\mathrm{mmHg})$ & $118.6 \pm 15.4$ & $118.8 \pm 15.5$ & $116.7 \pm 14.4$ & 0.06 \\
\hline Diastolic blood pressure $(\mathrm{mmHg})$ & $74.9 \pm 10.0$ & $75.0 \pm 9.9$ & $73.6 \pm 11.2$ & 0.06 \\
\hline Pulse rate (bpm) & $72.7 \pm 10.7$ & $72.7 \pm 10.8$ & $72.7 \pm 10.5$ & 0.99 \\
\hline \multicolumn{5}{|l|}{ Lifestyle } \\
\hline \multicolumn{5}{|l|}{ History of smoking } \\
\hline Current & $323 / 2727(11.8)$ & $293 / 2518(11.6)$ & $30(14.4)$ & 0.24 \\
\hline Former & $726 / 2727(26.6)$ & $674 / 2518(26.8)$ & $52(24.9)$ & 0.55 \\
\hline Never & $1678 / 2727(61.5)$ & $1551 / 2518(61.6)$ & $127(60.8)$ & 0.81 \\
\hline \multicolumn{5}{|l|}{ Comorbidities } \\
\hline Hypertension & $769(28.1)$ & $721(28.5)$ & $48(23.0)$ & 0.09 \\
\hline Diabetes mellitus & $190(6.9)$ & $174(6.9)$ & $16(7.7)$ & 0.67 \\
\hline Hypercholesterolemia & $834(30.4)$ & $782(30.9)$ & $52(24.9)$ & 0.07 \\
\hline Fatty liver & $338(12.3)$ & $320(12.6)$ & $18(8.6)$ & 0.09 \\
\hline Gout & $145(5.3)$ & $137(5.4)$ & $8(3.8)$ & 0.33 \\
\hline Metabolic syndrome & $336(12.3)$ & $315(12.5)$ & $21(10.0)$ & 0.31 \\
\hline Atrial fibrillation & $19(0.7)$ & $19(0.8)$ & $0(0)$ & 0.21 \\
\hline Anemia & $330(12.0)$ & $295(11.7)$ & $35(16.7)$ & 0.03 \\
\hline Previous CVD & $102(3.7)$ & $93(3.7)$ & $9(4.3)$ & 0.64 \\
\hline \multicolumn{5}{|l|}{ Laboratory data } \\
\hline Hemoglobin (g/dL) & $14.1 \pm 1.4$ & $14.2 \pm 1.3$ & $13.8 \pm 1.5$ & $<0.001$ \\
\hline BUN (mg/dL) & $14.1 \pm 3.4$ & $14.2 \pm 3.4$ & $13.6 \pm 3.8$ & 0.02 \\
\hline Creatinine (mg/dL) & $0.73 \pm 0.14$ & $0.74 \pm 0.14$ & $0.67 \pm 0.14$ & $<0.001$ \\
\hline eGFR $\left(\mathrm{mL} / \mathrm{min} / 1.73 \mathrm{~m}^{2}\right)$ & $77.5 \pm 12.9$ & $76.9 \pm 11.7$ & $85.4 \pm 21.4$ & $<0.001$ \\
\hline eGFR (last visit), $\left(\mathrm{mL} / \mathrm{min} / 1.73 \mathrm{~m}^{2}\right)$ & $74.9 \pm 13.4$ & $75.2 \pm 12.6$ & $71.5 \pm 20.4$ & 0.011 \\
\hline$\Delta \mathrm{eGFR}$ change $\left(\mathrm{mL} / \mathrm{min} / 1.73 \mathrm{~m}^{2}\right.$ year $)$ & $-0.83 \pm 3.63$ & $-0.21 \pm 2.79$ & $-8.41 \pm 4.0$ & $<0.001$ \\
\hline $\operatorname{HbA1c}(\%)$ & $5.7 \pm 0.5$ & $5.7 \pm 0.5$ & $5.7 \pm 0.7$ & 0.07 \\
\hline Total cholesterol $(\mathrm{mg} / \mathrm{dL})$ & $206.0 \pm 34.0$ & $206.5 \pm 33.9$ & $199.6 \pm 35.2$ & 0.005 \\
\hline LDL-C (mg/dL) & $125.5 \pm 30.5$ & $126.0 \pm 30.4$ & $120.2 \pm 32.2$ & 0.01 \\
\hline $\mathrm{HDL}-\mathrm{C}(\mathrm{mg} / \mathrm{dL})$ & $67.6 \pm 18.4$ & $67.8 \pm 18.4$ & $66.0 \pm 18.5$ & 0.18 \\
\hline Uric acid (mg/dL) & $5.5 \pm 1.3$ & $5.5 \pm 1.3$ & $5.4 \pm 1.4$ & 0.78 \\
\hline $\mathrm{CRP}(\mathrm{mg} / \mathrm{dL})$ & $0.11 \pm 0.45$ & $0.10 \pm 0.34$ & $0.21 \pm 1.10$ & 0.155 \\
\hline \multicolumn{5}{|l|}{ Urine test } \\
\hline Proteinuria & $28 / 2727(1.0)$ & $21 / 2518(0.8)$ & $7(3.3)$ & 0.001 \\
\hline
\end{tabular}

Data are expressed as mean \pm standard deviation or number (\%)

$e G F R$ estimated glomerular filtration rate, $B M I$ body mass index, $C V D$ cardiovascular disease, $B U N$ blood urea nitrogen, $L D L-C$ low density lipoprotein cholesterol, $H D L-C$ high density lipoprotein cholesterol, $C R P$ C-reactive protein 
Table 2 Determinants of eGFR decline

\begin{tabular}{lcll}
\hline Variable & $\mathrm{p}$ value & Odds ratio & $95 \% \mathrm{CI}$ \\
\hline Male gender & 0.26 & 0.84 & $0.62-1.14$ \\
Age $\geq 60$ years old & 0.55 & 0.92 & $0.69-1.22$ \\
Body mass index $>25 \mathrm{~kg} / \mathrm{m}^{2}$ & 0.96 & 0.99 & $0.70-1.42$ \\
Hypertension & 0.09 & 0.73 & $0.51-1.05$ \\
Diabetes mellitus & 0.45 & 1.24 & $0.71-2.16$ \\
Hypercholesterolemia & 0.17 & 0.79 & $0.56-1.11$ \\
Gout & 0.62 & 0.83 & $0.39-1.75$ \\
History of smoking & 0.51 & 1.11 & $0.82-1.50$ \\
eGFR at baseline $>75 \mathrm{~mL} /$ & $<0.001$ & 1.86 & $1.38-2.52$ \\
$\quad$ min/1.73 m & & & \\
CRP $>0.3$ mg/dL & 0.17 & 1.48 & $0.85-2.59$ \\
Anemia & 0.03 & 1.56 & $1.04-2.35$ \\
Proteinuria & 0.001 & 5.22 & $2.04-13.34$ \\
\hline
\end{tabular}

$C I$ confidence interval, eGFR estimated glomerular filtration rate, $C R P$ C-reactive protein $\mathrm{m}^{2}$ ), hypertension, DM, hypercholesterolemia, hyperuricemia, history of smoking, eGFR at baseline $(>75 \mathrm{~mL} /$ $\left.\mathrm{min} / 1.73 \mathrm{~m}^{2}\right)$, CRP $(>0.3 \mathrm{mg} / \mathrm{dL})$, anemia, and proteinuria showed that higher eGFR [odds ratio (OR), 1.86; $\mathrm{p}<0.001$ ], anemia (OR 1.56; $\mathrm{p}=0.03$ ) and proteinuria (OR 5.22; $\mathrm{p}=0.001$ ) were independent predictors of eGFR decline

In the "Results" section under the sub-heading "Subgroup analysis"

Study subjects were divided into two groups based on the presence of DM. The baseline characteristics in each group are shown in Appendix tables A/B. eGFR decline was seen in 16 of 190 subjects with DM (8.4\%) and in 193 of 2549 subjects without DM (7.6\%). In subjects with DM, anemia was not observed in subjects with eGFR decline, and therefore, OR of anemia for eGFR decline could not be calculated. Proteinuria tended to be associated with eGFR decline (OR 7.18; $p=0.09)$. In subjects without $D M$, anemia $(\mathrm{OR} 1.63 ; \mathrm{p}=0.02)$ and proteinuria (OR $4.44 ; \mathrm{p}=0.007)$ were associated with eGFR decline.

The corrected tables are given below. The conclusions of the published article are not affected.
Table 3 Multivariable analysis (subgroup analysis)

\begin{tabular}{lllllll}
\hline Variable & \multicolumn{2}{l}{ Diabetes mellitus absent } & & \multicolumn{2}{l}{ Diabetes mellitus present } & \\
\cline { 2 - 3 } & Adjusted OR (95\% CI) & & $\mathrm{p}$ value & & Adjusted OR (95\% CI) & p value \\
\hline Anemia & $1.63(1.08-2.46)$ & 0.02 & & $-(-)$ & & - \\
Proteinuria & $4.44(1.51-13.1)$ & 0.007 & & $7.18(0.76-68.2)$ & 0.09 \\
\hline
\end{tabular}

Adjusted for male, age $\geq 60$ years, BMI $>25 \mathrm{~kg} / \mathrm{m}^{2}$, hypertension, hypercholesterolemia, gout, history of smoking, eGFR at baseline $>75 \mathrm{~mL} / \mathrm{min} / 1.73 \mathrm{~m}^{2}, \mathrm{CRP}>0.3 \mathrm{mg} / \mathrm{dL}$

$C I$ confidence interval, $O R$ odds ratio

Appendix Table A: Subgroup analysis (DM present)

\begin{tabular}{|c|c|c|c|c|}
\hline Variable & $\begin{array}{l}\text { Overall } \\
(\mathrm{n}=190)\end{array}$ & $\begin{array}{l}\text { eGFR non-decline group } \\
(\mathrm{n}=174)\end{array}$ & $\begin{array}{l}\text { eGFR decline group } \\
(\mathrm{n}=16)\end{array}$ & $\mathrm{p}$ value \\
\hline Gender (male) & $107(56.3)$ & $99(56.9)$ & $8(50.0)$ & 0.60 \\
\hline Age (year) & $59.1 \pm 12.2$ & $59.4 \pm 12.5$ & $56.1 \pm 8.2$ & 0.29 \\
\hline $\mathrm{eGFR}\left(\mathrm{mL} / \mathrm{min} / 1.73 \mathrm{~m}^{2}\right)$ & $78.5 \pm 15.2$ & $78.5 \pm 15.2$ & $79.0 \pm 15.2$ & 0.89 \\
\hline eGFR (last visit), $\left(\mathrm{mL} / \mathrm{min} / 1.73 \mathrm{~m}^{2}\right)$ & $77.0 \pm 17.3$ & $78.2 \pm 17.1$ & $64.3 \pm 13.7$ & 0.001 \\
\hline$\Delta$ eGFR change $\left(\mathrm{mL} / \mathrm{min} / 1.73 \mathrm{~m}^{2}\right.$ year $)$ & $-0.05 \pm 4.20$ & $0.60 \pm 3.68$ & $-7.15 \pm 2.73$ & $<0.001$ \\
\hline Hemoglobin (g/dL) & $14.5 \pm 1.2$ & $14.6 \pm 1.3$ & $14.3 \pm 1.2$ & 0.47 \\
\hline Anemia & $9(4.7)$ & $9(5.2)$ & $0(0.0)$ & 0.35 \\
\hline Proteinuria & $6 / 188(3.2)$ & $4 / 172(2.3)$ & $2(12.5)$ & 0.03 \\
\hline
\end{tabular}

Data are expressed as mean \pm standard deviation or number (\%)

$D M$ diabetes mellitus, $e G F R$ estimated glomerular filtration rate 
Appendix Table B: Subgroup analysis (DM Absent)

\begin{tabular}{|c|c|c|c|c|}
\hline Variable & $\begin{array}{l}\text { Overall } \\
(\mathrm{n}=2549)\end{array}$ & $\begin{array}{l}\text { eGFR non-decline group } \\
(\mathrm{n}=2356)\end{array}$ & $\begin{array}{l}\text { eGFR decline group } \\
(\mathrm{n}=193)\end{array}$ & $\mathrm{p}$ value \\
\hline Gender (male) & $1412(55.4)$ & $1311(55.6)$ & $101(52.3)$ & 0.37 \\
\hline Age (year) & $58.8 \pm 11.7$ & $58.9 \pm 11.7$ & $58.7 \pm 11.7$ & 0.89 \\
\hline $\mathrm{eGFR}\left(\mathrm{mL} / \mathrm{min} / 1.73 \mathrm{~m}^{2}\right)$ & $77.4 \pm 12.8$ & $76.7 \pm 11.4$ & $86.0 \pm 21.7$ & $<0.001$ \\
\hline eGFR (last visit), (mL/min/1.73 $\left.\mathrm{m}^{2}\right)$ & $74.7 \pm 13.1$ & $74.9 \pm 12.2$ & $72.1 \pm 20.8$ & 0.003 \\
\hline$\Delta$ eGFR change $\left(\mathrm{mL} / \mathrm{min} / 1.73 \mathrm{~m}^{2}\right.$ year $)$ & $-0.89 \pm 3.58$ & $-0.27 \pm 2.71$ & $-8.51 \pm 4.09$ & $<0.001$ \\
\hline Hemoglobin $(\mathrm{g} / \mathrm{dL})$ & $14.1 \pm 1.4$ & $14.1 \pm 1.3$ & $13.7 \pm 1.5$ & $<0.001$ \\
\hline Anemia & $321(12.6)$ & $286(12.1)$ & $35(18.1)$ & 0.02 \\
\hline Proteinuria & $22 / 2539(0.9)$ & $17 / 2346(0.7)$ & $5(2.6)$ & 0.007 \\
\hline
\end{tabular}

Data are expressed as mean \pm standard deviation or number $(\%)$

$D M$ diabetes mellitus, $e G F R$ estimated glomerular filtration rate

Publisher's Note Springer Nature remains neutral with regard to jurisdictional claims in published maps and institutional affiliations. 